CASE NOTES

\title{
SPONTANEOUS HYPHAEMA WITH ABNORMAL IRIS VESSELS*
}

BY

\author{
P. U. FECHNER
}

Hamburg, Germany

A woman aged 42 was examined in the Casualty Department of the Royal Eye Hospital, London, at 8.30 p.m. on June 30, 1956.

She complained of slight blurring of right vision associated with a little pain which had begun 7 hours before.

This had never occurred previously. There was no history of physical strain or excitement, nor was there any relevant family or personal history concerning her ocular or general condition.

Examination.-Visual acuity was $6 / 9$ in the right eye and $6 / 6$ in the left eye uncorrected.

External examination of the right eye showed no abnormality. A fine stream of blood was squirting without pulsation into the aqueous humour from a small ovoid sac attached to the pupillary margin at 11 o'clock. This well-defined stream descended in the aqueous humour to form a thin layer of blood in the lower part of the anterior chamber. Although the stream was continuous the amount of blood in the anterior chamber did not increase, suggesting that it was being removed from the angle as quickly as it was collecting. Occasional slight irregularities in this flow caused "mushrooming" in its course (Figure).

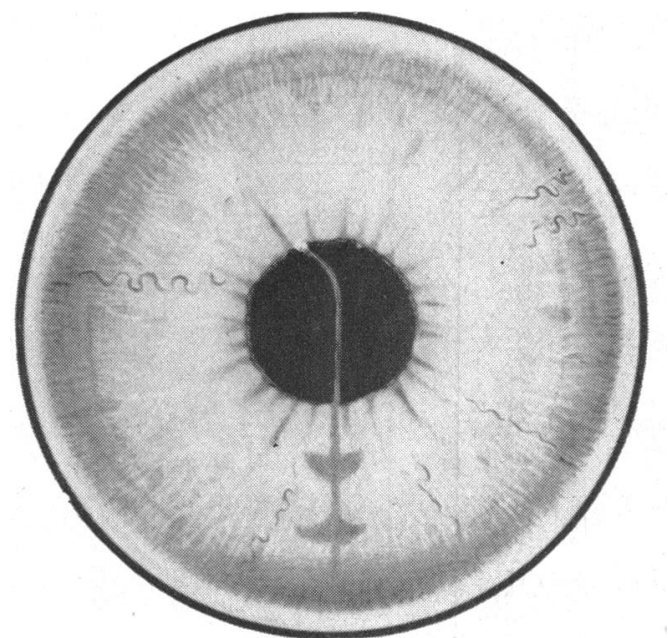

Figure.-Anterior segment of right eye. A small stream of blood is being ejected from a microaneurysm into the aqueous humour, "mushrooming" in its course and forming a thin hyphaema. Tortuosity of iris vessels is seen especially at 9 o'clock. The iris stroma is rather more loose than is apparent in the illustration.

* Received for publication April 12, 1957. 
Slit-lamp examination showed that the blood was being ejected from a small pale pink sac on the pupillary margin, the appearance of which strongly suggested a ruptured microaneurysm. Its size was about $0.15 \mathrm{~mm}$. There was a similar but smaller intact swelling at 1 o'clock on the margin.

The iris stroma was grey and loose and the iris vessels were clearly visible except at the sphincter. They were tortuous and slightly distended, especially one vessel in the 9 o'clock radius. Their appearance, in fact, resembled an acute inflammation, but there were no other signs of an inflammatory condition. At 10 o'clock on the surface near the margin there was a fine network of capillaries which showed the "cattle-truck" phenomenon.

The pupils were both approximately $2 \mathrm{~mm}$. in diameter and their reactions were normal.

The right lens and vitreous were normal. The fundus showed a small scar of old choroiditis near the disc which corresponded with a small steep-edged scotoma.

The left eye was normal apart from the iris, which showed the same scarcity of pigment and loose fibrillary structure as the right. The vessels in the iris stroma showed more marked tortuosity than in the right eye, especially those in the 6,9, and 11 o'clock radii, and gonioscopy revealed the 6 o'clock-vessel to emerge from a small conglomeration of loops in the angle of the anterior chamber. There was a small swelling on the pupillary margin at 1 o'clock similar to those in the right eye.

A placebo was prescribed and the patient was asked to return on the following morning when a small thrombus was seen attached to the sac at 11 o'clock in the right eye. One day later this thrombus had disappeared and all other signs of blood in the anterior chamber were gone. The vision in this eye was still 6/9 (corrected to 6/5) but no longer subjectively blurred.

A full investigation of the respiratory, digestive, and central and peripheral nervou; systems showed no abnormal findings; blood examinations (including clotting, prothrombin, and bleeding time, and capillary fragility) and urinary examinations were normal. In liver function tests, serum bilirubin, direct and indirect, alkaline phosphatase, and zinc sulphate turbidity were all within normal limits. At the first estimation, thymol turbidity showed 2 units and thymol flocculation was ++ . The second estimation showed 4 units and ++ respectively. (There was no history of hepatitis or jaundice and the significance of these slightly abnormal findings was never discovered.)

\section{Discussion}

This case is reported on account of its unusual iris vascular pattern with micro-aneurysms at the pupillary margins. No vascular anomalies were discovered elsewhere in the body and no disease processes were found to suggest an explanation for the unusual vessels. One therefore assumes a congenital origin.

Angiomatous malformations of the iris are rare (Duke-Elder, 1940). Rodin (1929) reviewed nine cases of alleged iris angioma and described one case of his own. Only three of the cases reviewed appeared to him to have been truly angiomatous, but all showed tumorous formation. This also applies to the cases described by Fuchs (1913) and to that reported by Knapp (1928).

The iris vascular pattern of the case presented here showed no indication of neoplastic origin. The tortuosity of the iris vessels, the loose fibrillary structure of the stroma of the iris, and the scarcity of iris pigment resembled the findings in the case reported by Tyson (1932), but the latter also 
presented a naevus flammeus of the face and globe associated with glaucoma and a calcified vascular growth of one occipital lobe of the brain with homonymous hemianopia on the contralateral side. As mentioned above no such signs of systemic abnormalities were found in the new case described in this paper.

\section{Summary}

A patient is described in whom the iris vascular pattern showed dilatation and tortuosity of some of the larger vessels. These abnormalities were associated with a fine capillary network near the right pupillary margin, and at least one micro-aneurysm on the right pupillary margin which ruptured and ejected a fine stream of blood into the aqueous humour.

I am indebted to Mr. T. M. Tyrrell for permission to publish this case, to Mr. T. A. S. Boyd for his help with preparing the text, and to Mr. T. Tarrant for his skilful preparation of the illustration.

\section{REFERENCES}

DUKe-ELder, S. (1940). “Text-book of Oṕhthalmology", vol. 3, p. 2456. Kimpton, London. FuCHS, E. (1913). v. Graefes Arch. Ophthal., 86, 155.

KNAPP, A. (1928). Arch. Ophthal. (N.Y.), 57, 219.

Rodin, F. H. (1929). Arch. Ophthal. (Chicago), $2,679$.

TYson, H. H. (1932). Ibid., 8, 365. 